\title{
Comparative analysis of multitouch interactive surfaces
}

\author{
Dawid Sielski, Wiktor Kozakiewicz, Michał Basiuras, Klaudia Greif, Jakub Santorek, \\ Przemysław Kucharski, Krzysztof Grudzień, Laurent Babout \\ Institute of Applied Computer Science, Łódź University of Technology \\ ul. Stefanowskiego 18/22, 90-924 Łódź, Poland
}

\begin{abstract}
The subject of this paper is to compare two different modality multi-touch interactive surfaces based on both: user experience and results of measurements in order to examine how different properties influence usefulness, in specific, their fitness to act as a "coffee table". Tests were conducted on the Microsoft PixelSense (AKA Surface) and a Samsung touch screen overlay both $40+$ inches diagonally. The study covers analysis of obtained measurements and summary of user experience collected over a number of summits and experiments. While tests for both devices returned very similar results, with the overlay more favorable, neither device could truly fit the tested use case due to their inconvenience, form factor and other issues.
\end{abstract}

\section{INTRODUCTION}

Touch enabled devices offer their users a fast and intuitive interface, and those properties skyrocketed the popularity of and demand for such solutions. Traditional control methods of electronic devices — in majority various buttons and switches have started to become less popular while touch solutions have become cheaper, more accurate and efficient [1]. To best facilitate people with this type of interface, it is crucial to well define human tendencies, perception, behavioral patterns, as well as what constitutes as "common sense", or intuition, while using them [2], [3].

\section{Technology And Methods}

\section{A. Technology}

There are 3 devices of importance in this paper. The Microsoft PixelSense (AKA. Microsoft Surface, SUR40), Samsung Touch Overlay (both being under tests) and Basler camera (for testing). SUR40 is a touchscreen table with a built in desktop computer. It uses infrared transmitters and sensors mounted underneath the 40" screen itself to complement a more conventional capacitive touchscreen. Such a system can detect many inputs and differ their shapes.

The Samsung Touch overlay is a frame that can be mounted on a 40" TV screen to turn it into a touch screen. The sides of the frame contain built in infrared transmitters and on opposite sides receivers are mounted. This system can detect up to 6 inputs but will not distinguish their shapes.
For testing purposes a Basler ACE Camera ACA2040 180KC was used to measure time between relevant events. The camera itself is capable of capturing 180 frames per second with a resolution of 2046 pixels by 2046 pixels, although in these tests the camera was set to capture only 100 frames per second, due to the screens' 60 hertz refresh rate. The lens that was used for the Basler camera was the Computar M2518-MPW2 with a focal length equal to $16 \mathrm{~mm}$, an iris range of $\mathrm{F} 2.0$, and a $2 / 3$ " format.

\section{B. Input lag and multi-touch capability test}

The test involved a Basler high-speed camera which was used to measure the interval between interaction with the screen and the device's reaction. A Google testing tool called cross touch latency was used. Two test were conducted. First using the click mode of the tool where a time stamp of the moment of the finger breaking contact with the touch area and a time stamp of the reaction (the screen going black) were captured via the highspeed camera. In the second test the reaction to a dragging action was assessed in a similar manner with the scroll mode. In both parts the camera was set to the side of a table looking at it in an angle to adequately distinguish the movement of the testing instrument while still being able to note the reaction of the testing tool.

In multi-touch capability test, the devices' ability to deal with multiple inputs was tested. This was done in three ways. First, using the Microsoft Paint application, which has multi-touch capability out of the box. During the test an increasing number of parallel lines was drawn at the same time. When one of the lines failed to be drawn, the test was considered as failed. The tests were repeated and accuracy changes noted. The second way involved devices such as phones. They were placed on the touch surface in random locations and the device's reaction was noted. The third way was a combination of the first and second, where a few phones were placed on the display and concurrently, parallel lines were drawn. With this, the device's ability to act as a "table" was tested. 


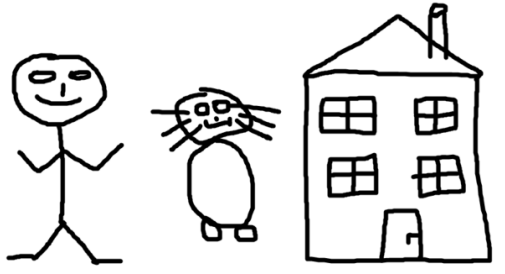

Figure 1. Examplary image to be recreated by the user.

\section{User study protocol}

There were $\mathrm{n}=18$ participants. The group consisted mostly of students who attend technical studies. At the beginning, users were introduced to both of the devices on which they were asked to perform 4 prepared tasks. Participants were only told that the devices are controlled by touch. All of them had the same tasks in the same order.

In the first task users were asked to find a weather forecast on the Internet and then shortly report the weather for today and tomorrow. This task made the user use the device in a way that one would use on a daily basis and allowed us to gather their first impressions. Second task required the users to use MS Paint in order to reproduce simple picture of a human, a house and a cat. The third task invited the user into the touch enabled game called "Angry Birds". This task gave users the opportunity to gather opinions about the accuracy or the response time of the touch screen. During the fourth task users had to rewrite first four lines from famous Polish novel. This task allowed user for comparison of speed and accuracy of input during relatively simple task of tapping proper keyboard buttons manifested on the screen via the on-screen keyboard. Picture that was presented to recreate can be viewed as Figure 1.

For each task, the participant was asked to perform it on one device and then again on the other device. After each try, the user would rate, in a scale from one to ten, how comfortable with the device they felt executing specified task. The next two questions asked if the task would have been easier or more comfortable if they had access to a mouse and keyboard, or if they were performing it on a regular smartphone.

\section{RESULTS}

Firstly, the input lag test provided information about time between user touching and e reaction visible for the user. During whole test twenty measures were performed from which it can be said that theirs response times are very close to each other because for the PixelSense it was $146.0 \mathrm{~ms}$ (std: 8.6 and median: 145) and for Touch Overlay it was $148.1 \mathrm{~ms}$ (std: 11.8 and median: 140).

Secondly, multi-touch capability test were performed for both PixelSense as well as for Touch

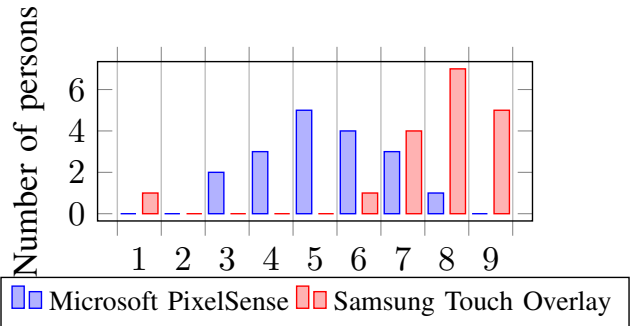

Figure 2. Users level of comfort while searching for information.

Overlay devices. For the first test, the PixelSense supported drawing up to 10 lines in Paint. After seven lines a noticeable loss of accuracy was recorded. As Paint only uses 10 touch points, the built-in Surface Shell was used for further testing. Up to 35 touch points were recorded as working before the size of the display made distinguishing any more difficult. Only objects with conductive surfaces could be used to interact with the screen. The screen would often recognize the palm of the hand as a touch point. For the second test, the PixelSense recognized the phones as touch points in the middle of their center of mass fairly well. Some devices, however, would not be recognized by the device, and some would cause glitches when put too close together. For the third test, the phones placed on PixelSense would correctly register as a touch point and would not otherwise interfere from drawing other parallel lines.

Next, Samsung Touch Overlay was tested. For the first test, the overlay supported up to six concurrent lines in Paint. Drawing them too close to each other caused accuracy problems. Items such as pens could be used to interact with the screen. For the second test, the overlay would not recognize the phone as a touch point, unless only a single corner of it was close to the screen at a time. For the third test, a placed phone or other obstruction would prevent any touch near it in a cross formation from registering.

\section{A. User study}

Results from the user study are presented in Fig. 2 - 5. Fig. 2 shows information about level of comfort during first task was presented. Moreover in this task the Touch Overlay got the better ending score because the average level of comfort was at 7.6 (std: 1.8, median: 8) than the PixelSense which got 5.3 (std: 1.4, median: 5).

During second test users were asked to replicate a simple drawing. The average level of comfort on Touch Overlay was at 6.3 (std: 2.0 , median: 7 ) and on the PixelSense at 6.1 (std: 2.1 , median: 7 ).

Additionally in this test users were asked to provide level of satisfaction after performing the drawing test. The results can be found in Figure 4 . 


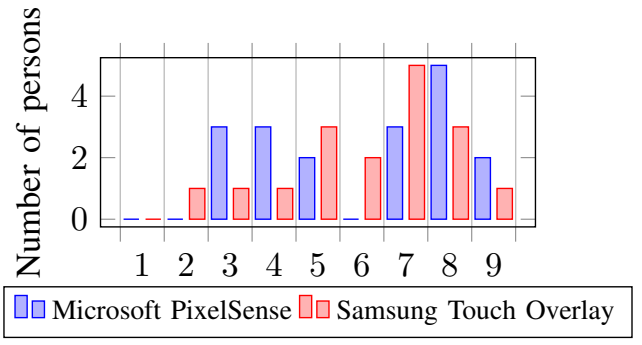

Figure 3. Users level of comfort while drawing in paint.

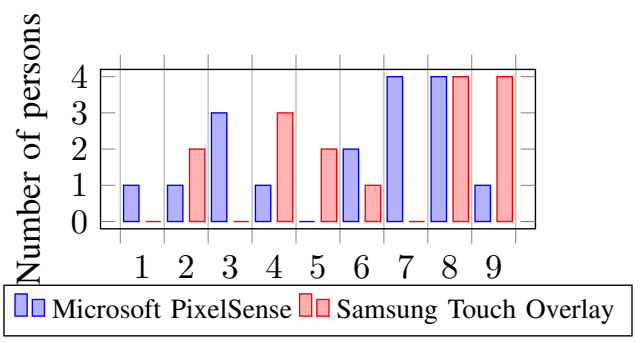

Figure 4. Users level of satisfaction on completing drawing in paint.

The summarized results are as follows. On Touch Overlay average score was at 6.3 (std: 2.5 , median: 7) and o n PixelSense at 5.0 (std: 2.5, median: 7).

The following test was focused on performance during the game "Angry Birds". Once more users graded their comfort during the test (the results can be seen on Figure 5), the following results were obtained. On the Touch Overlay average level of comfort was at 5.5 (std: 2.3, median: 6) and on PixelSense 5.1 (std: 2.0, median: 5)

Last part involved potential users in typing part of a text. The total results can be viewed in Figure 6. On Touch Overlay average was at 6.2 (std: 1.5, median: 6) and on PixelSense at 6.0 (std: 1.9, median: 6).

\section{DISCUSSION}

Tests on Input lag for both devices suggest a response time of around 150 milliseconds, while average response time of a human lands between 200 and 250 milliseconds. During user tests the lag did not however raise any complaints. Nonetheless, it is high enough to influence activities requiring

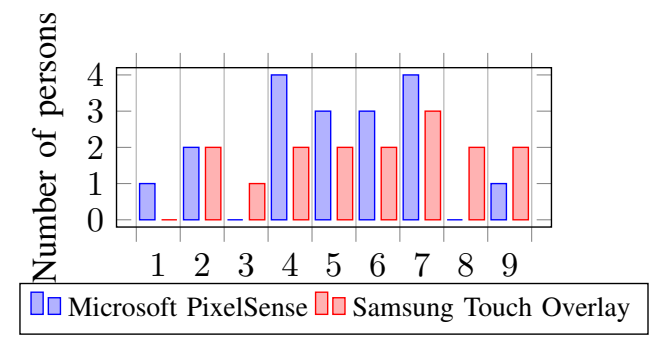

Figure 5. Users level of comfort while playing game (Angry Birds).

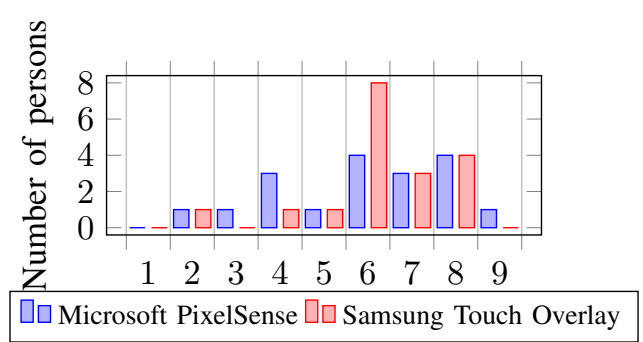

Figure 6. Users level of comfort while typing.

quick reaction times and this issue should be further investigated.

Multi-touch capability test was conducted in order to ascertain the viability of use by multiple users or with obstructions in form of phones or cups at the surface. While results indicate overperformance of PixelSense over Touch Overlay in handling objects placed on the surface, many people are already accustomed to using no more than 2 fingers to operate an interface. Hence, the overlay could be used by 3 people at once without issues, and as long as it is clear of any obstructions, could function as a coffee table. The biggest issues with such a configuration may in fact be the form factor of the overlay (no space to place hands) and the small viewing angle of the screen. On the other hand, the PixelSense, with a dedicated area for elbows and a great viewing angle, fails in a practical sense. The infrared touch supplement is extremely sensitive and frequently turns everything from the user's palm to a sleeve into a touch, resulting in less accuracy.

The first task of searching for the weather went well on both devices. Participants generally claimed that working with the Touch Overlay was easier than the PixelSense. Only a few judged the Touch Overlay as less accurate, in part because of the attachment of the device a few millimeters above the screen. Another issue that was brought up was the roughness of the digitizer not being fit for a touch device.

The painting exercise on PixelSense often required to inform participants about the infrared sensor position. After adjusting by moving the arm higher, the task could be completed, but not without comments that such a position is unnatural. The results of the task on both devices were satisfactory for most participants, who also noted that the device is more fit to it than a mouse or a phone. On the other hand users reported video game experience as much less satisfactory. Large number of participants could not reliably make 'hold and drag' motion and felt that whatever they succeed or not is random. During typing task many participants noted that the hand position required to use the touch keyboard was unusual and slowed 
them down. Standing up or adjusting the device's angle helped some of them.

Additionally, results from User study was applied to student $T$ test from which it can be stated that the averages of results from both devices are significant different $(\mathrm{t}=$ $2.496945, \mathrm{p}<0.014848$ )

\section{Future Work}

There exists a large swath of work that has to be done in the future to truly find out about themviability of large size touch screen devices in consumer situations. If they are to be installed as coffee tables, for example, a big amount of attention must be paid to the device's ability to deal with multiple users at the same time and to deal with objects placed on it in an intelligent way. However, even the best touch screen means nothing if users do not feel like they are enjoying an experience which is worth the money they spent, or at least one that is meaningfully different from the one they could have simply using a regular computer or smart-phone [4]. Thus, apart from research aimed to improve the hardware, research to find better use cases and create better suited applications is also needed. Also more devices should be tested [5] possibly with more than one person [6].

Also the field of persuasive systems could benefit from incorporating disputed technology [7]. It is interesting if multitouch devices of large screen, when widely available could support specific mundane tasks such as scientific image annotation by ordinary users, as proposed through online crowdsourcing systems recently [8] [9]. In fact there is a need of users of a process tomography domain for constructing a specialized system for presenting and visualising the raw and reconstructed measurement data such as available here [9] [8] [10] [11].

\section{Conclusions}

This paper shows a comparative study for two can be used with success for educational purposes [12]. modality interactive multitouch surfaces in terms of multi-user use. Both tested devices are clearly fit for the task of becoming a table in the living room, with the Samsung Overlay seemingly being the more convenient choice, yet generally users are less than eager to use these kind of devices because of their inconsistent touch experience. In order for them to be more popular, performance and usability should be improved in order for the users willing to switch from ordinary devices to multi-modal touch interactive surfaces. The devices were not versatile enough, with users quickly becoming disinterested after learning their flaws. Additionally, software support for multiple users is currently low, with few applications capable of handling more than one person (even two fingers only each). Another thing that prevent both devices from becoming a table is their inherent fragility and reluctancy of users to place objects on them. This technology, however, can be used with success for educational purposes [12].

\section{REFERENCES}

[1] C. Muller-Tomfelde and M. Fjeld, "Tabletops: Interactive Horizontal Displays for Ubiquitous Computing," Computer, vol. 45, pp. 78-81, 2012.

[2] A. Wojciechowski, K. Fornalczyk, "Camera navigation support in a virtual environment," Bulletin of the Polish Academy of SciencesTechnical Sciences, vol. 61, no. 4, pp. 871-884, 2015.

[3] J. Gerken, H.-C. Jetter, T. Schmidt, and H. Reiterer, "Can "touch" get annoying?" in ACM International Conference on Interactive Tabletops and Surfaces, ser. ITS '10. New York, NY, USA: ACM, 2010, pp. 257-258. [Online]. Available: http://doi.acm.org/10.1145/1936652. 1936704

[4] A. Wojciechowski and K. Fornalczyk, "Single web camera robust interactive eye-gaze tracking method," Bulletin of the Polish Academy of Sciences-Technical Sciences, vol. 63, no. 4, pp. 879-886, 2015.

[5] P. Kucharski, A. Romanowski, K. Grudzień, and P. Woźniak, "TomoSense: Towards Multi-Device Spatial Awareness Based on Independent Plane Sensing," in Cross Surface 2016 at ACM CHI'16, 2016.

[6] P. Wozniak, N. Goyal, P. Kucharski, L. Lischke, S. Mayer, and M. Fjeld, "RAMPARTS," in Proceedings of the 2016 CHI Conference on Human Factors in Computing Systems - CHI '16. New York, New York, USA: ACM Press, 2016, pp. 2447-2460. [Online]. Available: http://dl.acm.org/citation.cfm?doid=2858036.2858491

[7] P. Kucharski, P. Łuczak, I. Perenc, T. Jaworski, A. Romanowski, M. Obaid, and P. W. Woźniak, "A personal persuasive avatar for encouraging breaks in office work." Proceedings of the 2016 Federated Conference on Computer Science and Information Systems, vol. 8, p. 1627-1630, 2016. [Online]. Available: http://dx.doi.org/10. $15439 / 2016 \mathrm{~F} 491$

[8] I. Jelliti, A. Romanowski, and K. Grudzień, "Design of crowdsourcing system for analysis of gravitational flow using x-ray visualization," in Proceedings of the 2016 Federated Conference on Computer Science and Information Systems, ser. Annals of Computer Science and Information Systems, M. Ganzha, L. Maciaszek, and M. Paprzycki, Eds., vol. 8. IEEE, 2016, pp. 1613-1619. [Online]. Available: http://dx.doi.org/10.15439/2016F288

[9] C. Chen P. W. Woźniak, A. Romanowski, M. Obaid, T. Jaworski, J. Kucharski, K. Grudzień, S. Zhao, and M. Fjeld, "Using crowdsourcing for scientific analysis of industrial tomographic images," ACM Trans. Intell. Syst. Technol., vol. 7, no. 4, pp. 52:1-52:25, Jul. 2016 [Online]. Available: http://doi.acm.org/10.1145/2897370

[10] K. Grudzien, Z. Chaniecki, A. Romanowski, M. Niedostatkiewicz, and D. Sankowski, "Ect image analysis methods for shear zone measurements during silo discharging process," Chinese Journal of Chemical Engineering, vol. 20, no. 2, pp. 337 - 345, 2012. [Online]. Available:

http://www.sciencedirect.com/science/article/pii/S1004954112603966

[11] K. Grudzien, A. Romanowski, and R. A. Williams, "Application of a bayesian approach to the tomographic analysis of hopper flow," Particle and Particle Systems Characterization, vol. 22, no. 4, pp. 246253, 2005. [Online]. Available: http://dx.doi.org/10.1002/ppsc. 200500951

[12] P. Dillenbourg and M. Evans, "Interactive tabletops in education," International Journal of Computer-Supported Collaborative Learning, vol. 6, no. 4, pp. 491-514, 12 2011. [Online]. Available: http://link. springer.com/10.1007/s11412-011-9127-7 